\title{
On computing the physical sources of jet noise
}

Samuel Sinayoko, Anurag Agarwal

\section{Southampton \\ Institute of Sound and \\ Vibration Research}

$16^{\text {th }}$ AIAA Aeroacoustics Conference, Stockholm

7 June 2010 


\section{Introduction}

Objective

To understand the physical sources of jet noise. 


\section{Introduction}

Objective

To understand the physical sources of jet noise.

\section{Motivation}

- By-pass ratio is limited

- We need alternative strategies 


\section{Introduction}

Objective

To understand the physical sources of jet noise.

Motivation

- By-pass ratio is limited

- We need alternative strategies

\section{Methods}

- Goldstein's theory

- Direct Numerical Simulation 


\section{Part I}

\section{Theory}




\section{Sources definition}

$$
\mathrm{Nq}=0
$$

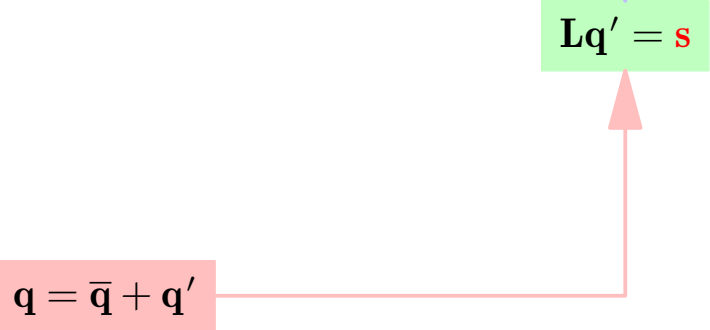




\section{Sources definition}

Physical sound sources

$$
f_{i}=-\frac{\partial}{\partial x_{j}}\left(\bar{\rho} \widetilde{v_{i}} \widetilde{v_{j}}\right)^{\prime}
$$

$$
\widetilde{v_{i}}=\overline{\rho v_{i}} / \bar{\rho}
$$




\section{Flow decomposition}

Space-time domain

$$
\overline{\mathbf{q}}=w * \mathbf{q}
$$

Wavenumber-frequency domain

$$
\overline{\mathrm{Q}}=\mathrm{W} \times \mathrm{Q}
$$




\section{Flow decomposition}

Space-time domain

$$
\overline{\mathbf{q}}=w * \mathbf{q}
$$

$$
\mathrm{q}(\mathrm{x}, \mathrm{t}) \stackrel{\text { F.T. }}{\longrightarrow} \mathrm{Q}(\mathbf{k}, \omega)
$$$$
\bar{q}(x, t) \longleftarrow \text { I.F.T. } \bar{Q}(k, w)
$$

Wavenumber-frequency domain

$$
\overline{\mathrm{Q}}=\mathrm{W} \times \mathrm{Q}
$$

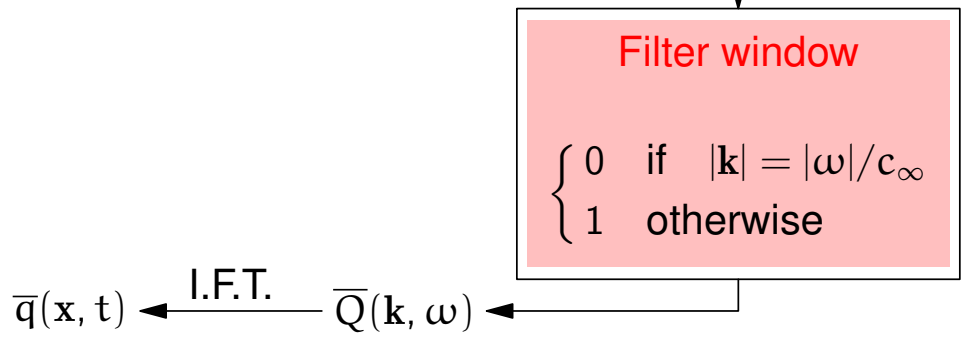


Part II

Numerical experiment 


\section{Mean flow}

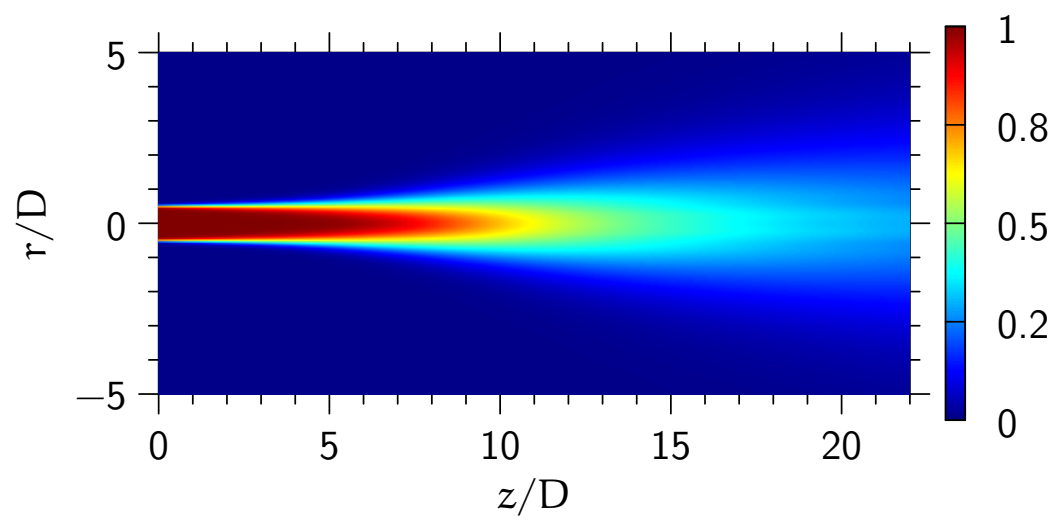

$$
\omega_{1}=2.2
$$

$$
\omega_{2}=3.4
$$




\section{Pressure snapshot}

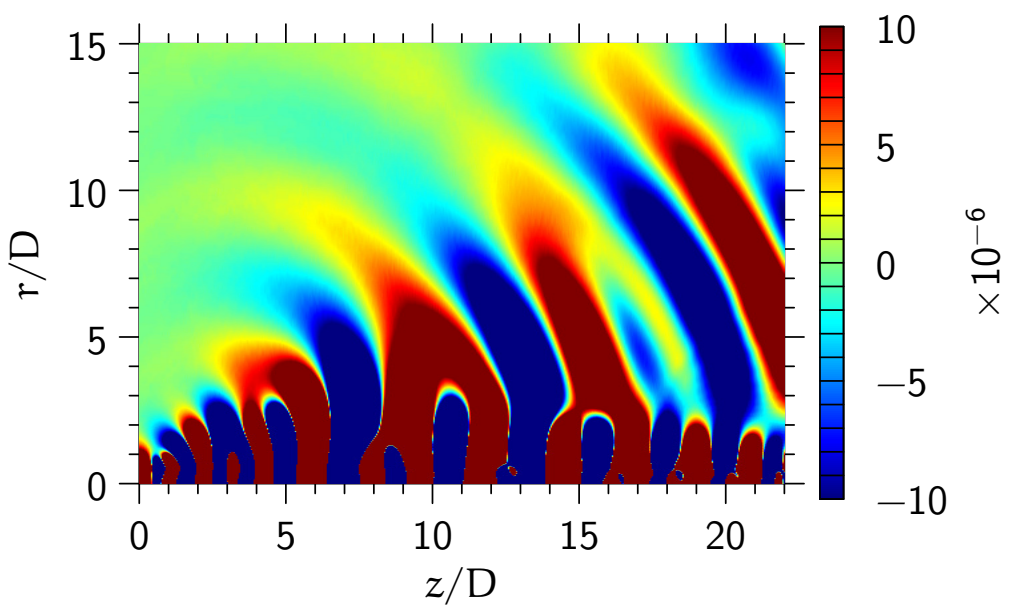



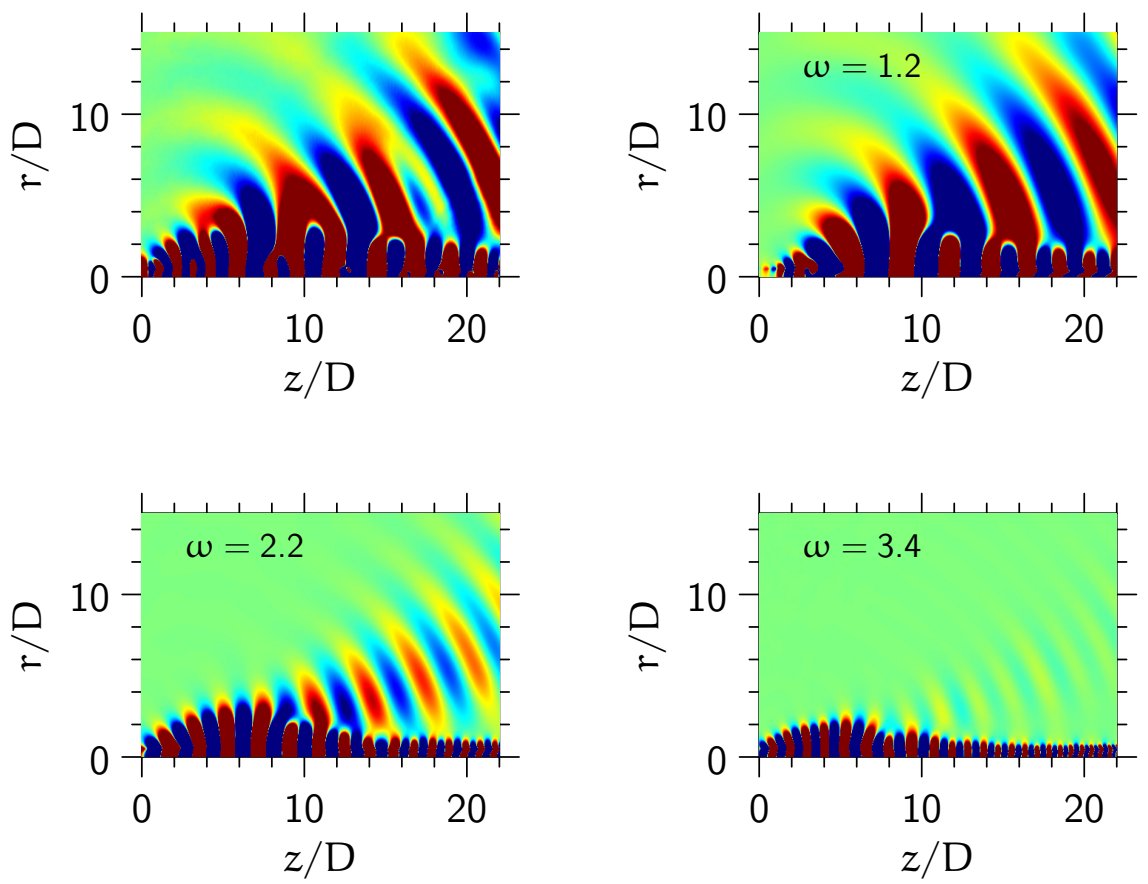


\section{Flow decomposition}

\section{Filter definition}

$$
\mathrm{k}_{\mathrm{ac}}=\Delta \omega / \mathrm{c}_{\infty}
$$

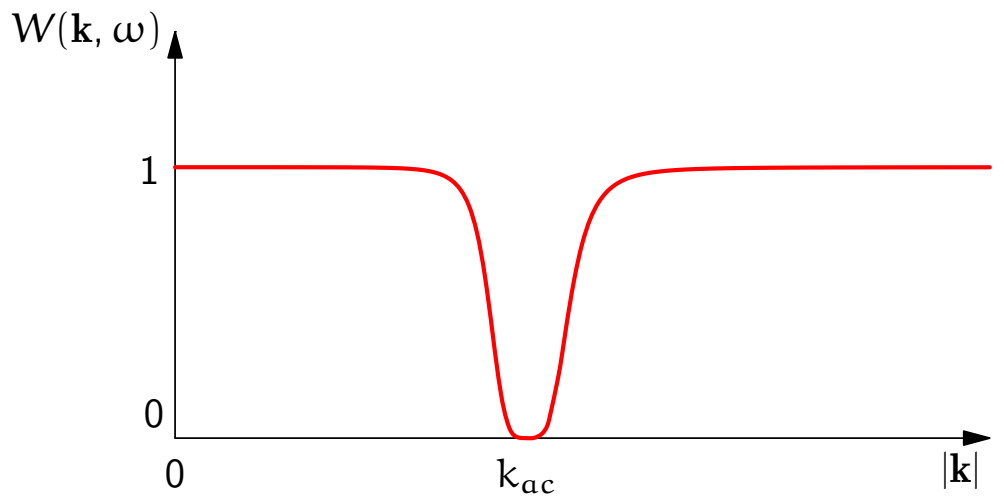




\section{Flow decomposition}

\section{Results}

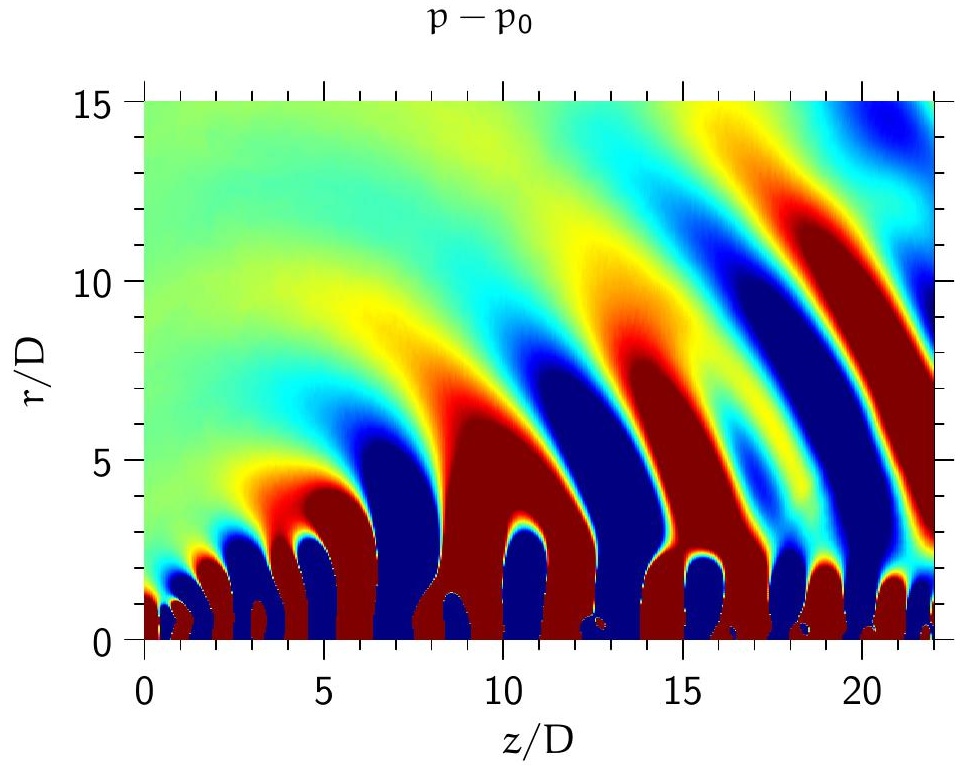




\section{Flow decomposition}

\section{Results}

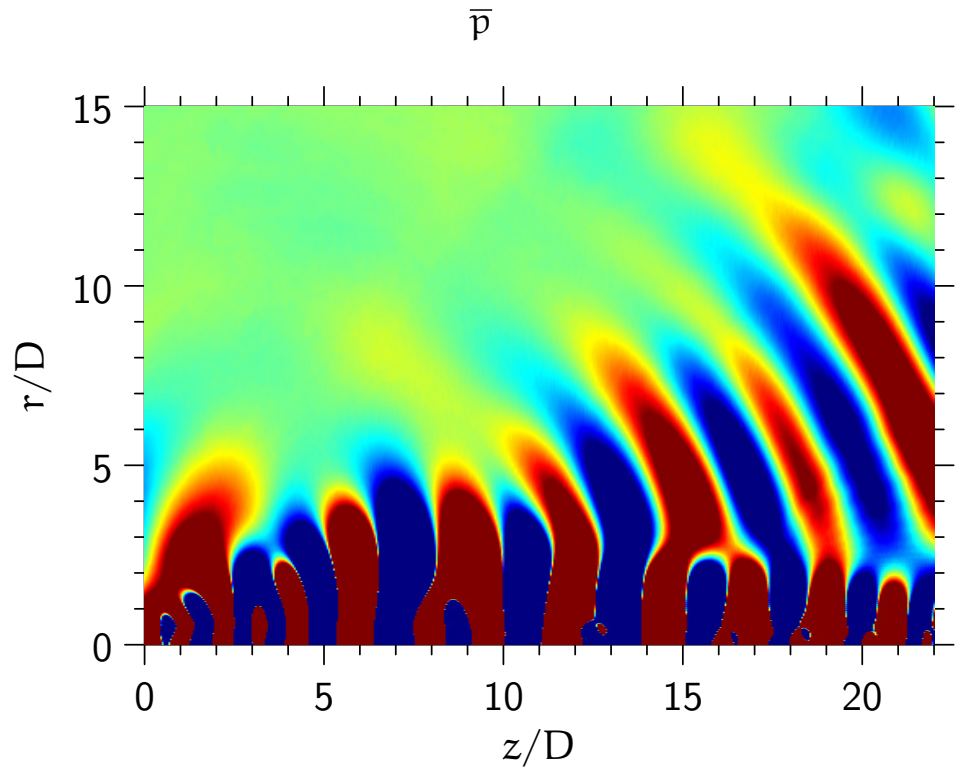




\section{Flow decomposition}

\section{Results}

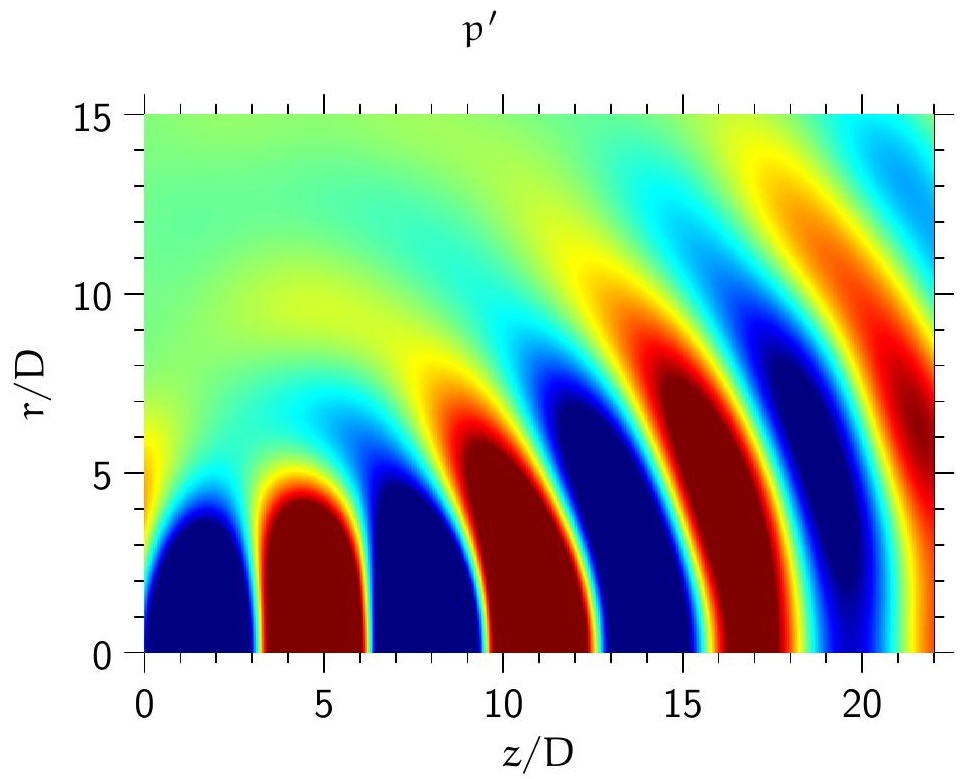




\section{Sound sources}

\section{Snapshot}

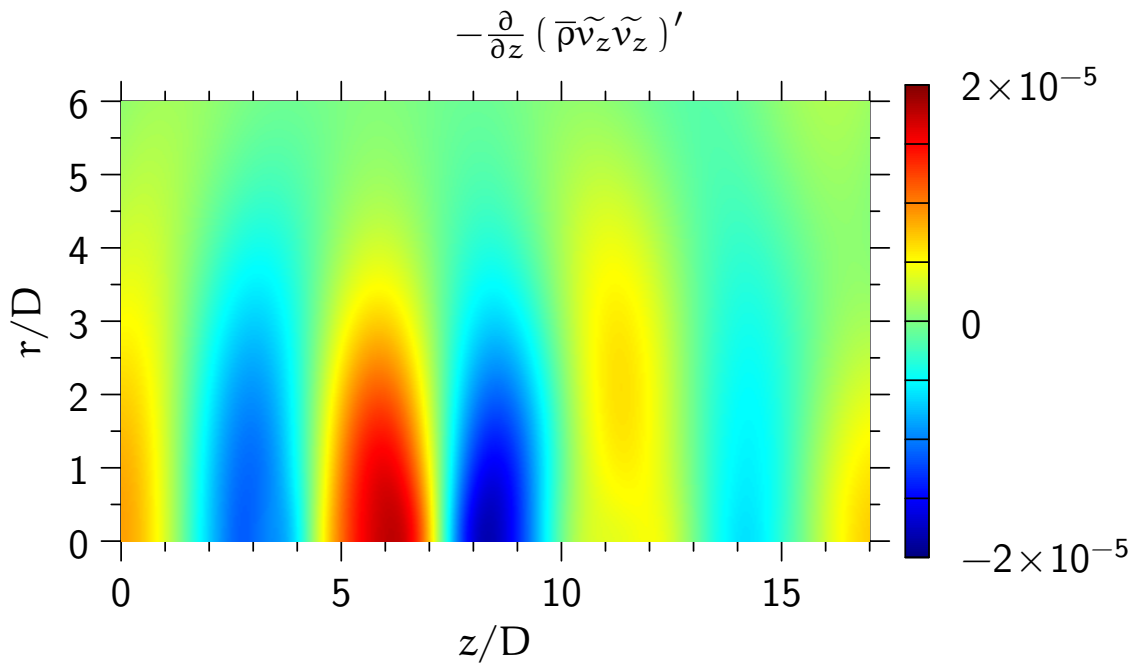




\section{Sound sources}

\section{Power spectum density}

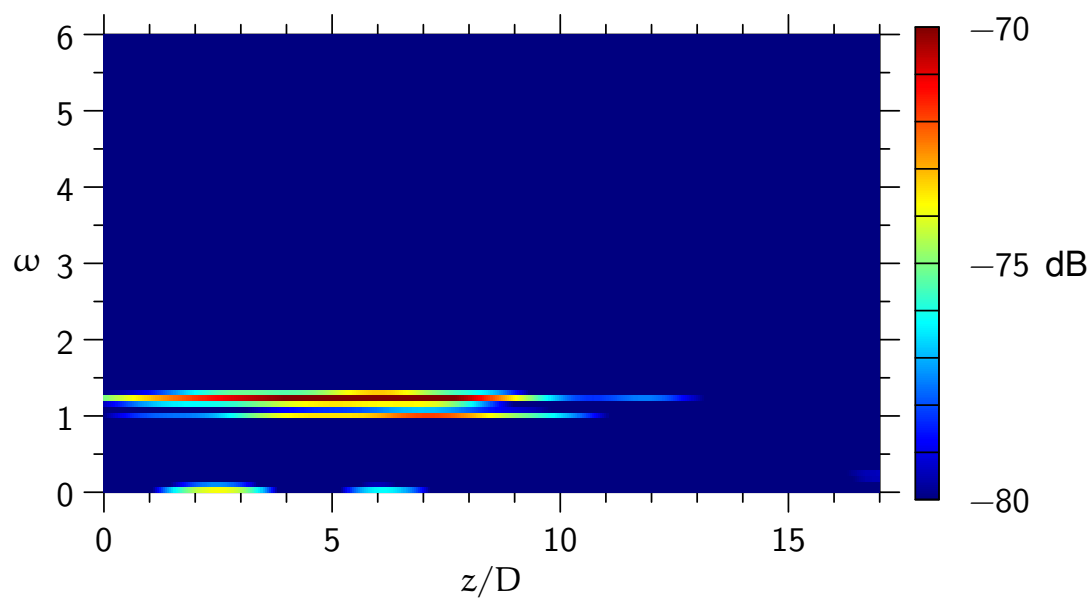




\section{Validation}

DNS

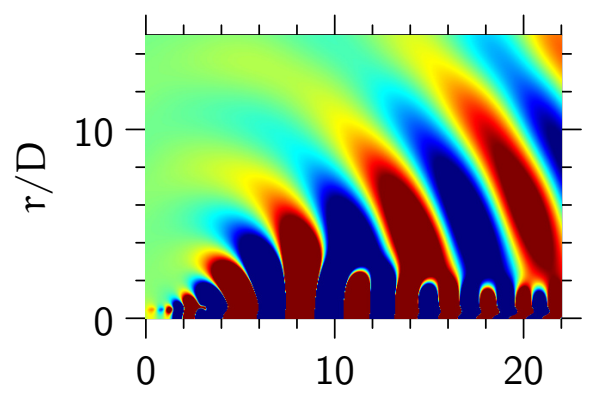

LEE

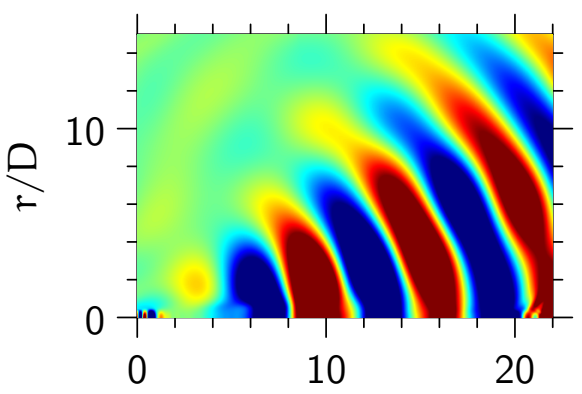


Validation

DNS

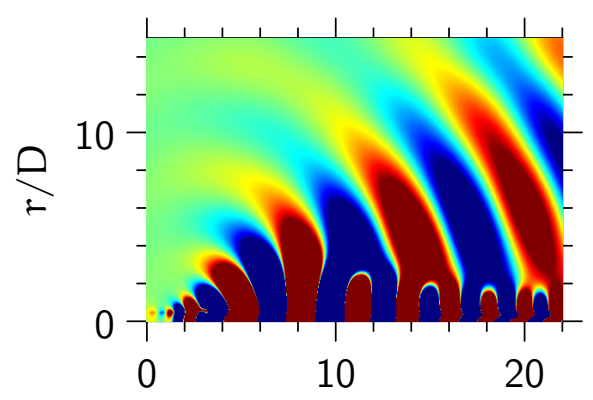

LEE
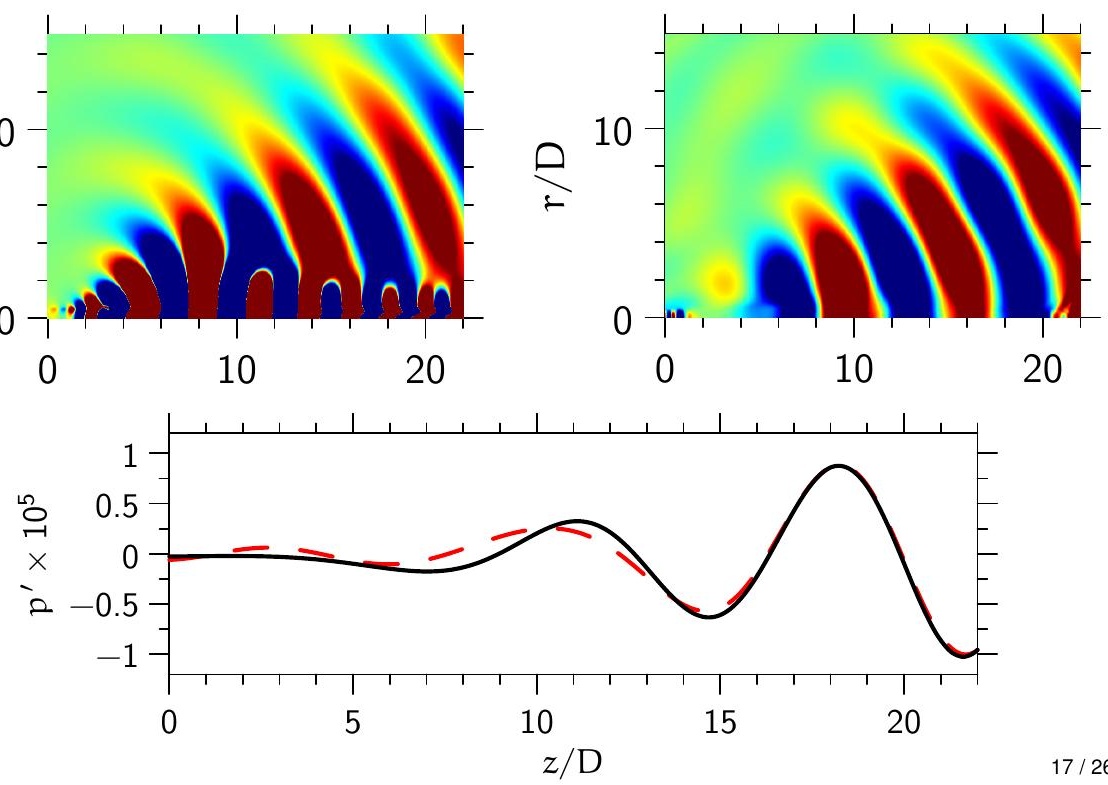


\section{Acoustic analogy sources}
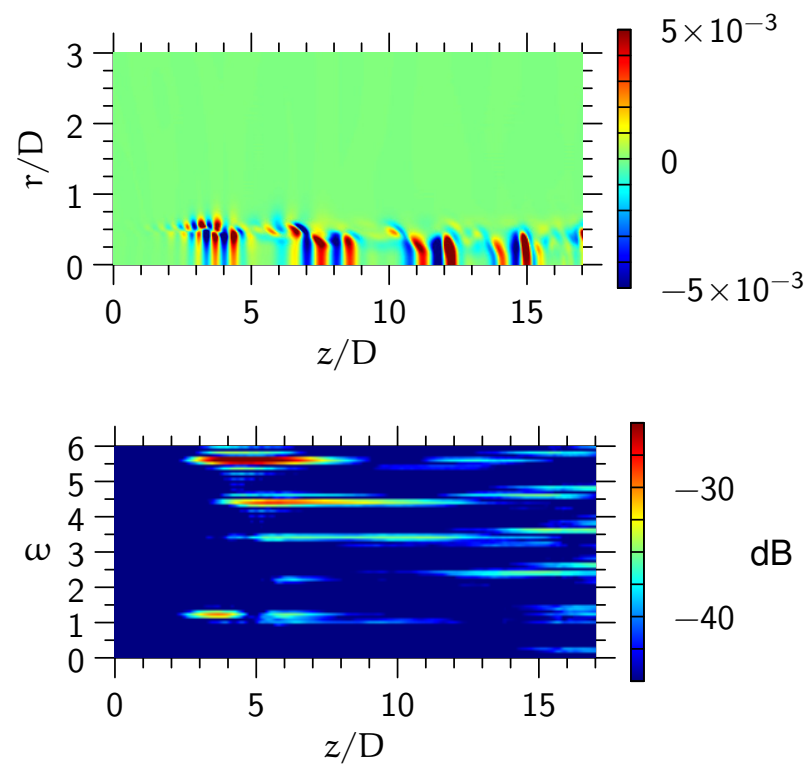


\section{Validation}

DNS

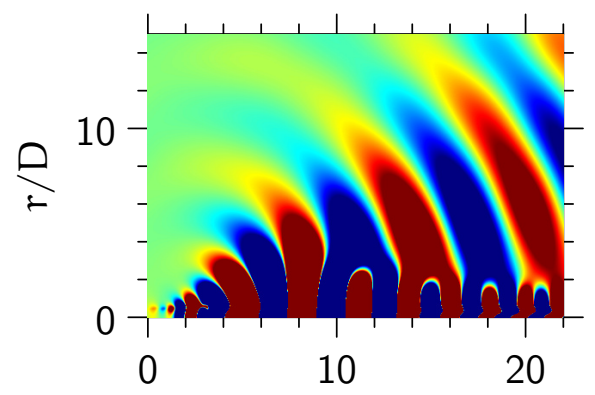

LEE

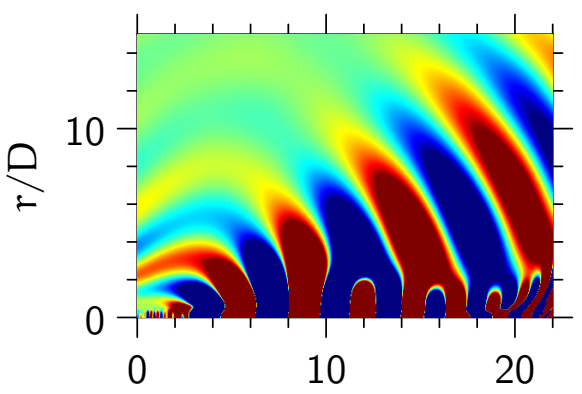


Validation

DNS

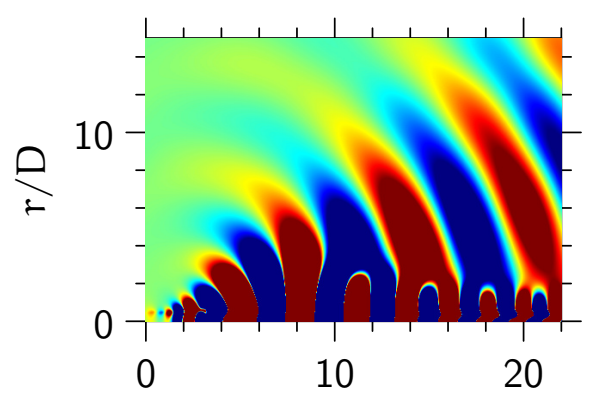

LEE
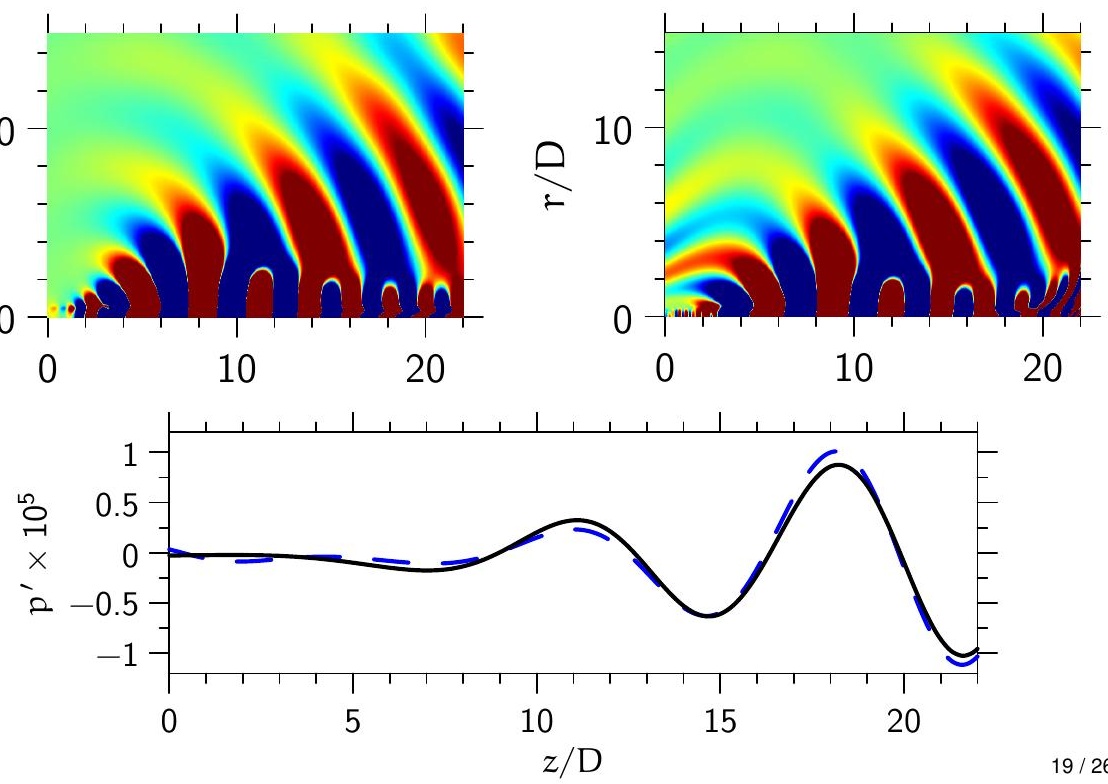


\section{Conclusion}

\section{Results}

- Use convolution filters

- Physical sound sources can be computed 


\section{Conclusion}

\section{Results}

- Use convolution filters

- Physical sound sources can be computed

\section{Future work}

- Model the sound sources

- Understand the physical mechanisms 


\section{Acknowledgements}

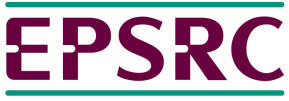

Engineering and Physical Sciences Research Council

Thank you! 


\section{Filtering of a two-dimensional shear layer}

Flow description

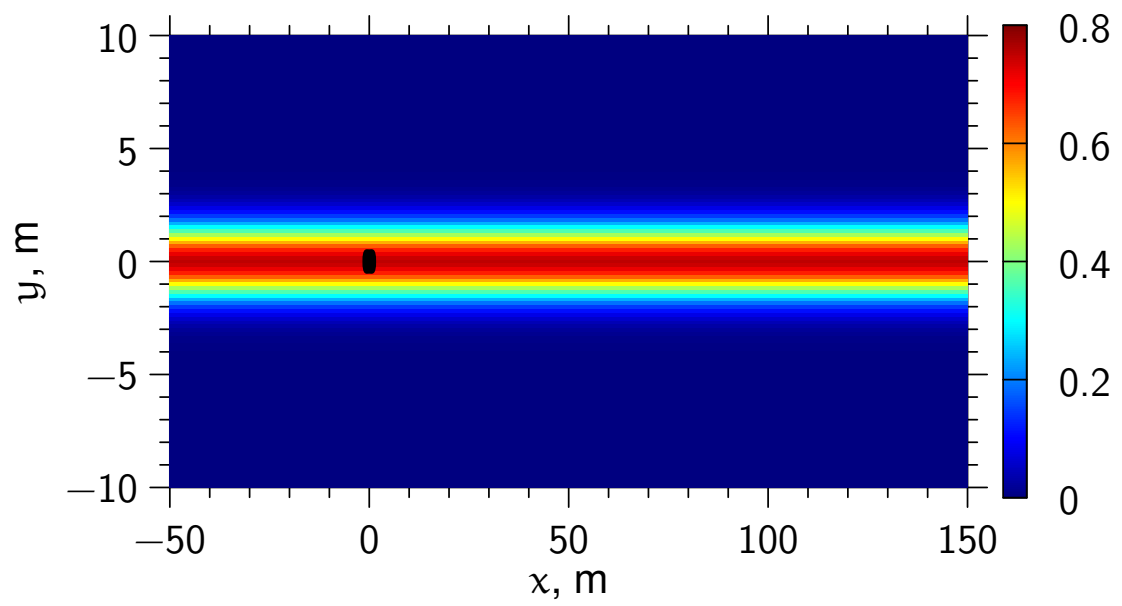




\section{Filtering of a two-dimensional shear layer problem}

\section{Pressure field}

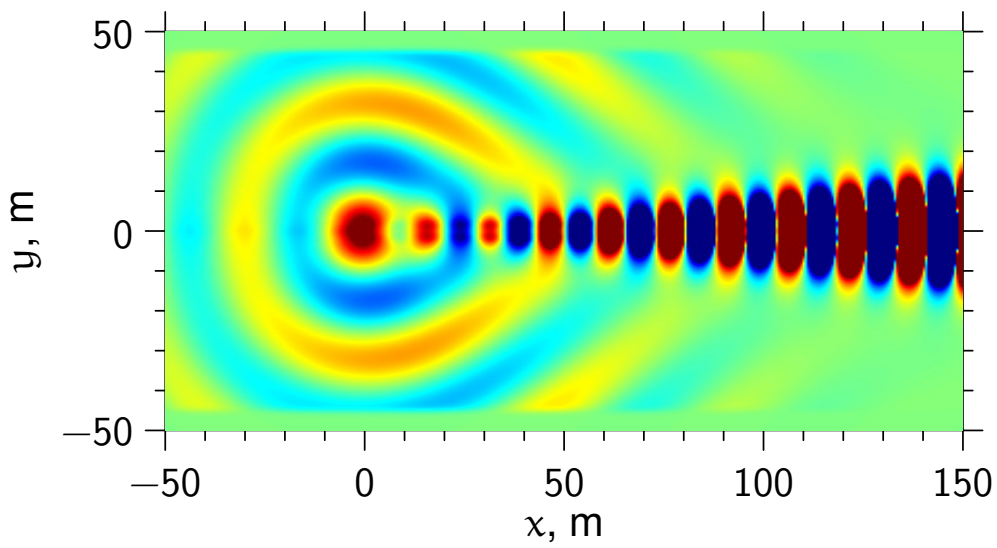




\section{Filtering of a two-dimensional shear layer}

Gaussian filter

$$
\begin{aligned}
& W(\mathbf{k}, \omega)=\exp \left(-\frac{\left(k_{x}-k_{0}\right)^{2}}{2 \sigma^{2}}\right)+\exp \left(-\frac{\left(k_{x}+k_{0}\right)^{2}}{2 \sigma^{2}}\right) \\
& k_{0}=0.41459, \quad \sigma=0.1
\end{aligned}
$$

Filter window

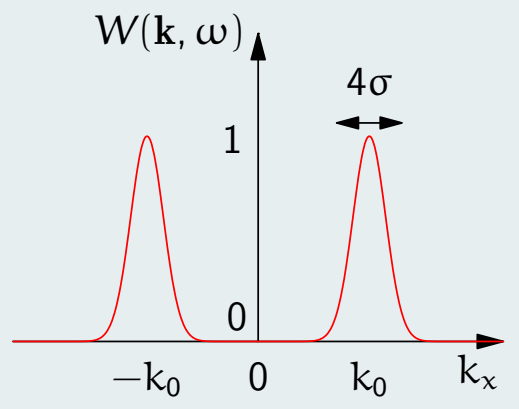

Fourier transform of pressure

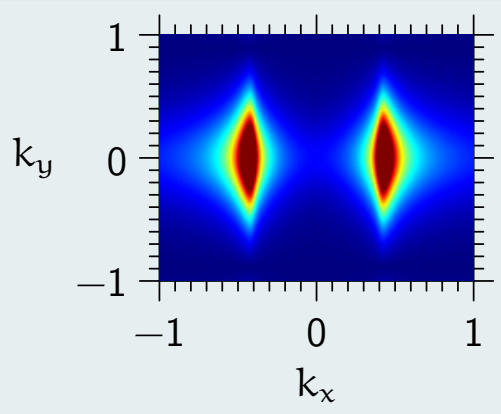




\section{Filtering of a two-dimensional shear layer}

\section{Results}

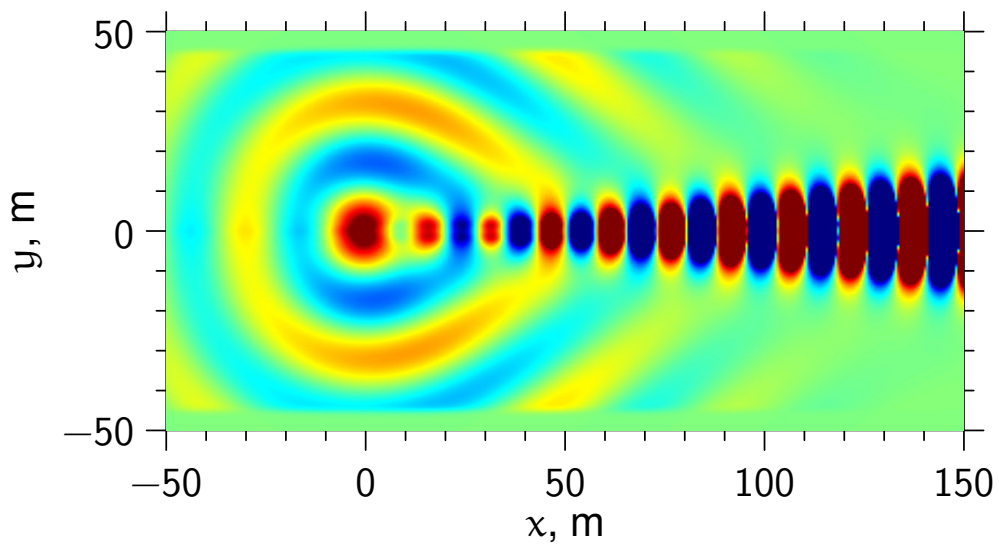


Filter of a two-dimensional shear layer problem Results

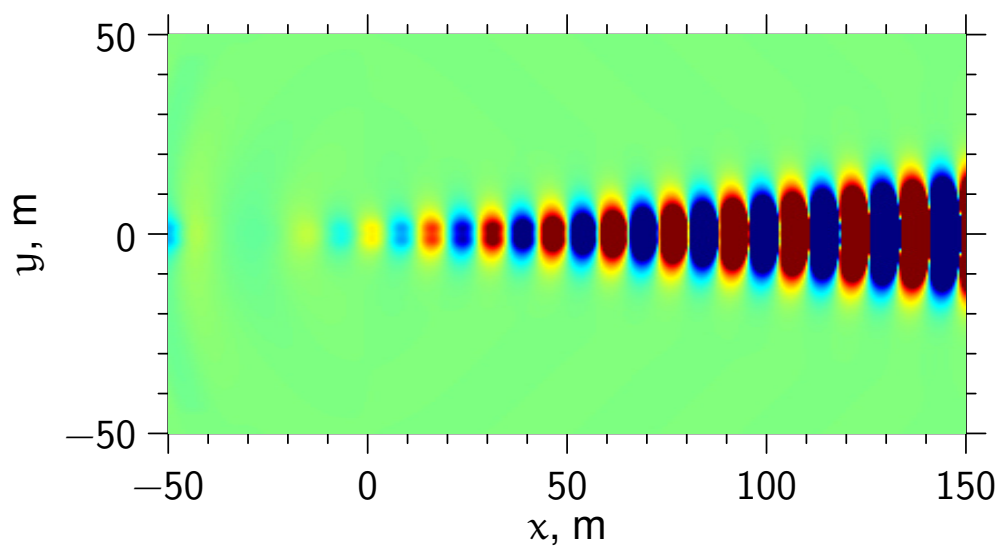

